\title{
EFFECT OF DIFFUSION ON PATTERNS IN EXCITABLE BELOUSOV-ZHABOTINSKII SYSTEMS
}

\author{
David A. KESSLER \\ Department of Physics, University of Michigan, Ann Arbor, MI 48109-1120, USA \\ Herbert LEVINE \\ Department of Physics and Institute for Nonlinear Science, University of California, San Diego, La Jolla, CA 92093, USA
}

Received 8 June 1988

Revised manuscript received 20 March 1989

Communicated by A.T. Winfree

\begin{abstract}
Travelling wave patterns occur frequently in chemically reacting systems; these include planar fronts, target patterns and spiral structures. We review the dispersion relation for planar waves, including the effects of diffusion in the "slow" field for a simplified piecewise-linear model, with a focus on the scaling behavior with respect to the relative rate of the "fast" and "slow" reactions. We discuss the implications of these results for spirals, showing the origins of Fife scaling and deriving a boundary-integral formulation of the spiral equations. We discuss the generalization to more realistic models, in particular, the popular Oregonator model for Belousov-Zhabotinskii rcactions.
\end{abstract}

\section{Introduction}

There has recently been an increasing amount of interest in the process of spatial pattern formation by chemically reacting systems, the best known of which is the Belousov-Zhabotinskii (BZ) reaction*. This interest has been spurred by sophisticated experiments [2] aimed at unraveling the dynamics of these patterns, as well as attempts at an analytical treatment.**

Depending on the reactant concentrations, the BZ system exhibits two types of behavior. The first is called oscillatory, and is characterized by

*For a review of the general phenomenology of the BZ reaction and travelling waves therein, see ref. [1].

**There have been many papers on chemical waves, too numerous to cite in detail. Our work has been motivated primarily by the efforts of Keener and Tyson, refs. [6, 10] below and Fife, ref. [11] below. spatially uniform spontaneous oscillations. The other type of behavior is generically referred to as excitable. Simply put, excitable kinetics means that the reacting system is locally stable but that small but finite perturbations can excite the system to perform large traversals in phase space. In a well-stirred reactor, the concentrations of reactants would rapidly equilibrate; in an open system, though, disturbances which "excite" the reaction can propagate through the material in the form of travelling waves. In a two-dimensional layer, possible wave patterns are planes, expanding circles (targets) or rotating spirals.

It is relatively easy to demonstrate that periodic trains [3] of planar reaction fronts can exist at a continuous range of velocities; we will see this later in the specific model we study here. Therefore, a complete solution to the planar problem consists of finding a dispersion relation between 
wavelength and velocity. Unfortunately, for complex reaction kinetics, even this simple level of analysis is only feasible numerically.

Target patterns are the next more complicated behavior exhibited by $\mathrm{BZ}$ reactions [4]. Here, the frequency and wavelength of the pattern are related by the dispersion relation for planar solutions, but the particular wavelength seen is dependent on the details of the pacemaker region [5] in which the parameters of the system are perturbed into the oscillatory regime.

The most interesting problem lies in the pattern selection problem for spiral wave patterns [6]. Here again, the frequency and wavelength of the pattern are related by the planar dispersion relation, as the pattern far from the spiral core is just a periodic planar travelling wave train. Experimentally, there appears to be a unique spiral pattern for given parameter values. This problem is reminiscent of the pattern selection problems in other cases of steady-state nonequilibrium dynamics, e.g. dendritic growth [7] and viscous fingering [8]. It has recently been determined that in both of these systems the experimentally realized pattern selection is explained by the fact that there is a unique stable steady-state solution [9]. It is unknown, however, if there is similarly a unique steady-state spiral solution to the $\mathrm{BZ}$ equations of motion that would fix the frequency and wavelength independently.

Experimentally, spiral structures possess a core region where the outgoing wavefront and waveback meet - see schematic given in fig. 1. It is certainly the case that diffusion of the "slow" field

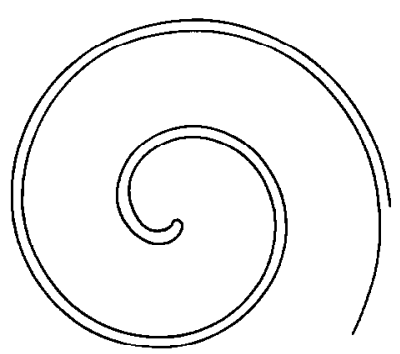

Fig. 1. Sketch of a BZ spiral wave pattern. plays a role in determining the core structure; it is unknown whether it is also relevant for the far distance outgoing waves. Keener and Tyson [6] have recently suggested a picture where the velocity of the almost planar fronts at large radial distances is sufficiently large so as to allow for the neglect of diffusive effects. They then impose a boundary condition at a "fictitious" inner radius so as to determine a unique rotating pattern. They further suggest that diffusion might fix this inner radius giving rise to a truly unique spiral, but this is only a conjecture. Fife [11], on the other hand, has suggested a different scaling for spiral waves. The relationship between these two proposals has yet to be clarified. It seems to us that progress towards resolving the issue of whether there is or is not a unique spiral solution requires a better understanding of diffusive effects.

The purpose of this paper is to analyze the effects of diffusion of the "slow" field in BZ-type systems, and its implications for the nature of spiral wave patterns. An important first step in this process has been taken recently by Dockery, Keener, and Tyson (DKT) [10]. In this paper, they calculated the dispersion relation for periodic travelling wave solutions, including the effects of diffusion of the "slow field." As these travelling wave solutions determine the asymptotics of the spiral solution at large distances from the spiral core, they provide important clues as to the role of diffusion in spirals.

We thus shall start by reviewing and expanding upon the work of DKT. As in ref. [10], we shall work in the context of a two-reactant system in which the ratio of the two reactions is governed by the small parameter $\epsilon$. For small $\epsilon$ the dynamics of the "fast" ficld can be eliminated, resulting in a two-phase problem for the "slow" field with a sharp interface dividing the two phases. The resulting field equations for the "slow" field are nonlinear and therefore difficult to analyze. As pointed out by DKT, it is convenient to introduce a simplified version of the "slow" field dynamics, in which the "slow" field satisfies a (different) linear equation in each phase. This simplified, 
piecewise-linear model (PLM) appears to capture the essential features of the problem, while allowing an analytic discussion of the dispersion relation.

In our presentation, we will focus in particular on how the scaling with $\epsilon$ changes as the velocity is varied. We shall see that there are generically three regions in parameter space. For very large velocity, diffusion is unimportant; for moderate velocities, diffusion effects set in and we demonstrate explicitly that the dispersion relation obeys a scaling originally suggested by Fife [11]. If $\epsilon$ is not sufficiently small, the Fife scaling will break down at small velocities.

We then examine the implications of these results for spiral wave patterns. We argue that, in the small-e limit, there are two distinct possibilities for the $\epsilon$ dependence of the pattern. In particular, if one assumes that the asymptotic velocity of the pattern is slow enough that diffusion effects are important even at large distances, the pattern then scales uniformly in $\epsilon$ in the small- $\epsilon$ limit. Appropriate rescalings then yield the $\epsilon$-independent equation first derived by Fife.

A crucial feature of the Fife regime is that the "slow" field is everywhere near the critical value for which the interface velocity (determined by the value of the "slow" field on the interface) has zero velocity. This implies that if the Fife scaling hypothesis is correct, the Fife equations for the spiral are valid in the small $\epsilon$ limit for an arbitrary choice of kinetic equations, and not just for the simplified PLM. The underlying kinetics would then just serve to fix some parameters in the Fife equation. We present a calculation of these parameters for the popular Oregonator model of $\mathrm{BZ}$ kinetics. More important, however, is the fact that the resulting spiral dynamics would be "universal", independent of the details of the underlying dynamics.

One of the crucial ingredients in the solution of the velocity selection problems in dendritic growth and viscous fingering, from both an analytical as well as a numerical standpoint, has been the reformulation of the problem, via a Green's function boundary-integral technique, into an integro-differential equation for the interface [12]. The linearity of the PLM allows us to formulate a boundary-integral equation for the spiral wave pattern for this model. This recasting of the problem should greatly simplify the task of numerically solving the equation and determining whether there is a unique solution. As this reformulation can be carried out at arbitrary $\epsilon$, the method, if successful is potentially capable of testing the validity of the Fife scaling ansatz. The boundaryintegral formulation should also prove invaluable in performing a stability analysis of the numerically determined solution(s).

We view the results contained in this work as a necessary first step towards the resolution of the velocity selection problem for spirals. At the very least, our discussion of the boundaries between different scaling (in $\epsilon$ ) regimes should serve to clarify some of the disagreement in the literature on this subject.

\section{Periodic traveling waves}

\subsection{Preliminaries}

In this section, we discuss the periodic traveling wave solutions in BZ-type dynamics. This problem has been previously studied by Dockery, Keener and Tyson [10] (DKT), but we shall reproduce their results herein both in order to set notation as well as to make the paper self-contained. Our goal here is to emphasize the points that will be important for the rest of our paper.

The general model we will consider is a tworeaction system of the form:

$\epsilon \dot{u}=\epsilon^{2} \nabla^{2} u+f(u, v)$,
$\dot{v}=\epsilon D \nabla^{2} v+g(u, v)$.

Here, $\epsilon$ is the ratio of the reaction rates for the two species, $u$ and $v . D$ is the ratio of the diffusion constants for the two species, assumed to be 


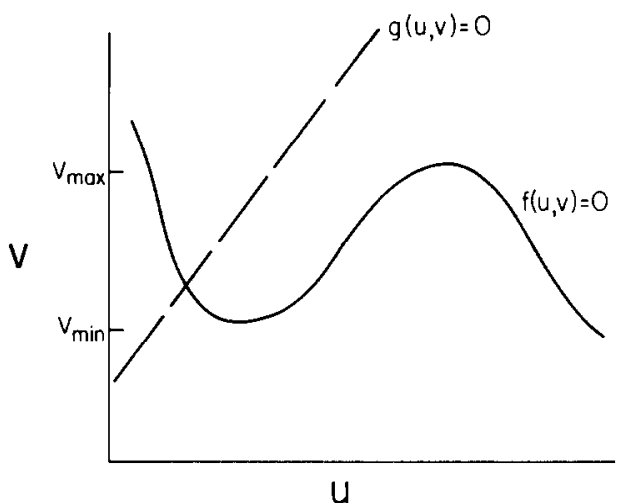

Fig. 2. Typical nullclines for the functions $f, g$.

of order 1 . We have used the traditional scaling for the space coordinate in which the diffusion constant for the $u$ field is taken to be $\epsilon$. The functions $f$ and $g$ have nullclines of the general form indicated in fig. 2 , so that for a given value of $v$ in some range $v_{\min }<v<v_{\max }$, there are 3 zeros of $f(u, v)$. We may denote these zeros by $u_{0}(v), u_{ \pm}(v)$, with $u_{-}(v)<u_{0}(v)<u_{+}(v)$. We are particularly interested in the limit of small, positive $\epsilon$, where there is a sharp interface between the $u>u_{0}(v)$ and $u<u_{0}(v)$ regions.

We will concern ourselves in this section with steady-state planar solutions propagating with some constant velocity $c$. We therefore transform to the comoving frame, whereupon the equations become

$$
\begin{aligned}
& \epsilon^{2} u^{\prime \prime}+\epsilon c u^{\prime}+f(u, v)=0, \\
& \epsilon D v^{\prime \prime}+c v^{\prime}+g(u, v)=0
\end{aligned}
$$

As is well known $[6,11]$ for small $\epsilon$ these equations essentially decouple. The equation for $u$ can be solved directly in this limit, yielding regions of slow variation in which $u$ is algebraically related to $v$, separated by regions of fast variation, of width $\approx \epsilon^{-1}$. In the "slow" regions, $u$ is given by either $u_{-}(v)$ or $u_{+}(v)$ while in the "fast" region, $u$ switches solution branch over a short distance and $v$ can be taken as constant. Examining the $u$ equation in the transition region yields a ( $\epsilon$-independent) relationship between the velocity $c$ of this soliton-like solution and the value of $v$ in the transition region. For a transition from $u$ to $u_{+}$with increasing $x$ we get

$v=\nu(c)$,

while for the reverse transition

$v=\bar{\nu}(c) \equiv \nu(-c)$

The equation for $v$ can then be solved treating the transition region as an infinitely thin interface between two "phases" in each of which $u$ is a different known function of $v$. The relation (2.3) serves, along with the continuity of $v$ and its first derivative across the interface, as the boundary conditions determining the dynamics of the interface. The resulting equation of motion for $v$ is in general nonlinear and can only be analyzed numerically.

It is to circumvent this roadblock that DKT introduced the expedient of replacing this nonlinear equation for $v$ with a different linear equation in each of the two phases. One may justify this piecewise-linear model (PLM) from several different perspectives. On one level, one can view it as a toy model, with which to illustrate in an analytic fashion the generic features of the problem. On the other hand, as pointed out by DKT, one can view the PLM as a first approximation to the full problem. In this case, we can derive the PLM by expanding the equation of motion for $v$ about some particular value. It will prove useful to choose to expand about $v_{\mathrm{s}}$, the value of $v$ for which the interface has zero velocity: $v_{\mathrm{s}} \equiv \nu(0)=$ $\bar{\nu}(0)$. In this approximation, the equation of motion for $v$ then has the form [10]

$\epsilon D v^{\prime \prime}+c v^{\prime}-b_{ \pm}\left(v-v_{\mathrm{s}}\right) \pm a_{ \pm}=0$.

It is interesting to note that the PLM is exact for a model in which the functions $f$ and $g$ are linear in $v$ and piecewise-linear in $u$ [13]. Then, due to the linearity, one can eliminate the $u$ field exactly, for any value of $\epsilon$, deriving a fourth-order piecewise-linear equation of motion for $v$. This 
allows one to investigate the accuracy of the small $\epsilon$ decoupling approximation and explore the effects of finite interface width in a convenient manner, while still only having to deal with one (linear) field.

As was discussed in the introduction, we are particularly interested in periodic solutions [3] of (2.4), with an alternating sequence of + and phases. Let us denote the width of the $+(-)$ regions by $x_{+}\left(x_{-}\right)$, choosing the point $x=0$ to lie on the interface between the - and + phases. There will then be interfaces between + and - at $-x_{-}$and $x_{+}$, which points are to be identified due to the periodic boundary conditions. In the + phase, $0<x<x_{+}$,

$v_{+}=v_{\mathrm{s}}+\frac{a_{+}}{b_{+}}+\alpha_{1} \exp \left(k_{1} x\right)+\alpha_{2} \exp \left(k_{2} x\right)$

and similarly, in the - phase, $-x_{-}<x<0$,

$v_{-}=v_{\mathrm{s}}-\frac{a_{-}}{b_{-}}+\alpha_{3} \exp \left(k_{3} x\right)+\alpha_{4} \exp \left(k_{4} x\right)$.

The $k_{1,2}$ are given by

$k_{1,2}=\frac{-c \mp \sqrt{c^{2}+4 \epsilon D b_{+}}}{2 \epsilon D}$

with analogous expressions for $k_{3}$ and $k_{4}$. At the interface $v$ and $v^{\prime}$ are continuous, with $v$ determined by the velocity $c$, yielding

$$
\begin{aligned}
& v_{+}(0)=v_{-}(0)=\nu(c) \equiv v_{0}, \\
& v_{+}^{\prime}(0)=v_{-}^{\prime}(0), \\
& v_{+}\left(x_{+}\right)=v_{-}\left(-x_{-}\right)=\bar{\nu}(c) \equiv \bar{v}_{0}, \\
& v_{+}^{\prime}\left(x_{+}\right)=v_{-}^{\prime}\left(-x_{-}\right) .
\end{aligned}
$$

This gives a system of six equations for the six unknowns, $\alpha_{1,2,3,4}$ and $x_{ \pm}$.

Now, generically $c \sim \mathrm{O}(1)$, so that (2.6) simplifies to $k_{1,3} \approx-c / \epsilon D, k_{2,4} \approx b_{ \pm} / c$. The largeness of $k_{1,3}$ implies that, assuming $x_{ \pm}$are not too small, $\alpha_{1} \exp \left(k_{1} x_{+}\right)$and $\alpha_{3}$ are exponentially small. Then, to lowest order in $\epsilon D / c^{2}$, we can solve (2.7) directly, giving

$$
\begin{aligned}
& \alpha_{1}, \alpha_{3} \exp \left(-k_{3} x_{-}\right) \sim \mathrm{O}(\epsilon), \\
& \alpha_{2}=v_{0}-v_{\mathrm{s}}-\frac{a_{+}}{b_{+}}, \\
& \alpha_{4}=v_{0}-v_{\mathrm{s}}+\frac{a_{-}}{b_{-}}, \\
& x_{+}=\frac{c}{b_{+}} \ln \left(\frac{a_{+}+b_{+}\left(v_{\mathrm{s}}-\bar{v}_{0}\right)}{a_{+}-b_{+}\left(v_{0}-v_{\mathrm{s}}\right)}\right), \\
& x_{-}=\frac{c}{b_{-}} \ln \left(\frac{a_{-}+b_{-}\left(v_{0}-v_{\mathrm{s}}\right)}{a_{-}-b_{-}\left(v_{\mathrm{s}}-\bar{v}_{0}\right)}\right) .
\end{aligned}
$$

Notice that this is precisely the result we would obtain if we set $D=0$, dropping the diffusion term in the $v$ equation (2.4). Of course, the lower order $\alpha_{1,3}$ terms generate narrow $(\sim \mathrm{O}(1 / \epsilon))$ boundary layers at the interfaces which are responsible for the continuity of $v^{\prime}$, which is lost if the diffusion term is dropped altogether. As these boundary layers are of the same width as the interface, however, the higher order analysis is nontrivial.

\subsection{The Fife regime}

We have just seen how the diffusionless limit arises from velocities $c \sim \mathrm{O}(1)$. We now investigate where this approximation breaks down. We derive the above result for the diffusionless limit by assuming $\exp \left(k_{1} x_{+}\right), \exp \left(k_{3} x_{-}\right) \ll 1$, or equivalently, $c x_{+} / \epsilon D \gg 1$. Examining (2.8) we see that $c \ll 1$ is necessary for this condition to be violated. For small $c$, we may approximate $v_{0}-v_{\mathrm{s}}$ $\approx \nu^{\prime}(0) c$. The smallness of this quantity allows the expansion of the logarithms in (2.8), implying $x_{ \pm} \propto c^{2}$. From (2.4) it is clear that diffusion effects first become important when $c x_{+} \sim \epsilon$, which translates to $c^{3} \sim \epsilon$, or $c \sim \mathrm{O}\left(\epsilon^{1 / 3}\right), x_{ \pm} \sim \mathrm{O}\left(\epsilon^{2 / 3}\right)$. One should notice that this range of $c$ is still sufficiently large that the approximations for $k_{i}$ in the previous section are still valid.

In this regime, first identified by Fife [11], we can solve (2.7) via a different approximation. Here, $k_{2} x_{+}, k_{4} x_{-} \sim \mathrm{O}\left(\epsilon^{1 / 3}\right)$, so we can expand $\exp \left(k_{2} x_{+}\right), \exp \left(k_{4} x_{-}\right)$. Setting $c=\epsilon^{1 / 3} \tilde{c}, x_{ \pm}=$ 
$\epsilon^{2 / 3} \tilde{x}_{ \pm}$we find at 0 th order in $\epsilon$

$\alpha_{1}=\alpha_{2}=0, \quad \alpha_{2}=-\frac{a_{+}}{b_{+}}, \quad \alpha_{4}=\frac{a_{-}}{b_{-}}$.

Solving (2.7) to next order we find, after some tedious algebra, that $\tilde{x}_{-}$is proportional to $\tilde{x}_{+}[10]$

$\tilde{x}_{-.}=\frac{a_{+}}{a_{-}} \tilde{x}_{+}$,

and $\tilde{x}_{+}$satisfies

$$
\begin{aligned}
& {\left[a_{+} \tilde{c} \tilde{x}_{+}-2 \nu^{\prime}(0) \tilde{c}^{3}\right] \sinh \frac{\tilde{c}}{2 D}\left(1+\frac{a_{+}}{a_{-}}\right) \tilde{x}_{+}} \\
& \quad=2 D\left(a_{+}+a_{-}\right) \sinh \frac{\tilde{c}}{2 D} \tilde{x}_{+} \sinh \frac{\tilde{c}}{2 D} \frac{a_{+}}{a_{-}} \tilde{x}_{+} .
\end{aligned}
$$

Eq. (2.11) implies that $\tilde{x}_{+}$, and so also $\tilde{x}_{-}$, monotonically decreases with $\tilde{c}$. For large $\tilde{c}, \tilde{x}_{+} \rightarrow$ $2 \nu^{\prime}(0) \tilde{c}^{2} / a_{ \pm}$, or $x_{ \pm} \rightarrow 2 \nu^{\prime}(0) c^{2} / a_{ \pm}$which matches on to the small-c limit of the result (2.8) for the diffusionless regime. At small $\tilde{c}$ [10],

$\tilde{x}_{ \pm}^{3} \rightarrow \frac{24 D^{2} \nu^{\prime}(0) a_{\mp}}{a_{ \pm}^{2}}$

so $\tilde{x}_{ \pm}$have nonzero limits as $\tilde{c}$ goes to 0 . The Fife regime thus interpolates between the relatively large velocity regime, $c \sim \mathrm{O}(1)$, where $x_{ \pm}$are also $\mathrm{O}(1)$, and a region where $x_{ \pm}$are small, $\mathrm{O}\left(\epsilon^{2 / 3}\right)$.

It is important to note for later that in the Fife regime, $v-v_{\mathrm{s}} \ll 1$ not only at the interphase boundaries but everywhere. This is due fundamentally to the smallness of $k_{2,4} x_{ \pm}$in this regime. Thus, we can equivalently obtain the Fife limit directly from the differential equation for $v,(2.4)$, by dropping the $v-v_{\mathrm{s}}$ term as can be verified explicitly.

\subsection{The stall $(c \rightarrow 0)$ limit}

There is one other limit which is possible to treat analytically, the stall limit, $c \rightarrow 0$. Now, as $c$ decreases, $k_{2,4}$ increase and so we would expect that the Fife approximation becomes less accurate. However, since in the Fife regime $x_{ \pm}$$\mathrm{O}\left(\epsilon^{2 / 3}\right)$ and even at $c=0, k_{2,4}$ is no larger than $\mathrm{O}\left(\epsilon^{-1 / 2}\right)$, it appears that the Fife approximation $k_{2,4} x_{ \pm} \ll 1$ is still valid down to $c=0$ for small $\epsilon$. As we shall see, this is indeed the case for small enough $\epsilon$, (how small being a function of the parameters $\left.b_{ \pm}, a_{ \pm}\right)$. The restriction of "small enough" will turn out to be surprisingly stringent, however, and not simply $\epsilon \ll 1$. Thus the Fife approximation is not valid in general for $c \ll \epsilon^{1 / 3}$.

In treating the $c \rightarrow 0$ limit, we notice that at lowest order in $c$, the solution is actually undetermined, with solutions for a continuous range of $x_{+}^{s}$. (We append a superscript $s$ to remind the reader that we are calculating for the stall solution, $c=0_{+}$.) For a given $x_{+}^{5}$, the solution is

$$
\begin{aligned}
\alpha_{1} & =\alpha_{2} \exp \left(-\sqrt{b_{+} / \epsilon D} x_{+}^{\mathrm{s}}\right) \\
& =-\frac{a_{+}}{b_{+}} \frac{1}{1+\exp \left(\sqrt{b_{+} / \epsilon D} x_{+}^{\mathrm{s}}\right)}, \\
\alpha_{3} & =\alpha_{4} \exp \left(\sqrt{b_{-} / \epsilon D} x_{-}^{\mathrm{s}}\right) \\
& =\frac{a_{-}}{b_{-}} \frac{1}{1+\exp \left(-\sqrt{b_{-} / \epsilon D} x_{-}^{\mathrm{s}}\right)},
\end{aligned}
$$

with $x_{-}^{\text {s }}$ given by [10]

$\tanh \frac{1}{2} \sqrt{\frac{b_{-}}{\epsilon D}} x_{-}^{\mathrm{s}}=\frac{a_{+}}{a_{-}} \sqrt{\frac{b_{-}}{b_{+}}} \tanh \frac{1}{2} \sqrt{\frac{b_{+}}{\epsilon D}} x_{+}^{\mathrm{s}}$.

At next order, a solvability condition emerges, yielding

$$
\begin{aligned}
& \frac{a_{+}}{b_{+}}\left(1-\frac{\sqrt{b_{+} / \epsilon D} x_{+}^{\mathrm{s}}}{\sinh \left(\sqrt{b_{+} / \epsilon D} x_{+}^{\mathrm{s}}\right)}\right) \\
& +\frac{a_{-}}{b_{-}}\left(1-\frac{\sqrt{b_{-} / \epsilon D} x_{-}^{\mathrm{s}}}{\sinh \left(\sqrt{b-/ \epsilon D} x_{-}^{\mathrm{s}}\right)}\right) \\
& =\frac{2 \nu^{\prime}(0) \sqrt{b_{+} \epsilon D}}{\tanh \left(\frac{1}{2} \sqrt{b_{+} / \epsilon D} x_{+}^{\mathrm{s}}\right)}\left(1+\frac{a_{-}}{a_{+}}\right) \text {. }
\end{aligned}
$$


For $\epsilon$ small enough, we note that $x_{+}^{s} \ll 1$ and we can solve these equations. In this limit, the equation relating $x_{-}^{\mathrm{s}}$ to $x_{+}^{\mathrm{s}},(2.13 \mathrm{~b})$, reduces to the simple proportionality of the Fife limit, (2.10). Furthermore, eq. (2.14) then also reproduces the result (2.12) for $x_{ \pm}$in the Fife limit. In other words, as advertised, the Fife regime extends all the way down to $c=0$ in the $\epsilon \rightarrow 0$ limit. For finite $\epsilon$, however, at $c \rightarrow 0, x_{ \pm}^{\mathrm{s}}$ approach nonzero values different from those predicted by the Fife approximation, (2.12). Comparing (2.10) and (2.13b), it is clear that the quality of the Fife approximation is controlled by the smallness of $x_{ \pm}^{\mathrm{s}}$, which is turn is controlled by the smallness of $\epsilon$. As $\epsilon$ is taken larger, for fixed $b_{+}, a_{+}$, the Fife approximation becomes worse. In fact, the stall solution disappears [10] above a critical value of $\boldsymbol{\epsilon}$, in which case the Fife approximation is very bad indeed! We can see this by noting that, from (2.13b), there is a maximum value that $x_{+}^{s}$ can attain, namely

$x_{+}^{s, \max }=2 \sqrt{\epsilon D / b_{+}} \tanh ^{-1}\left(a-\sqrt{b_{+}} / a_{+} \sqrt{b_{-}}\right)$,

at which point $x_{-}^{\text {s }}$ goes to $\infty$. (We assume here that $a_{-} \sqrt{b_{+}} / a_{+} \sqrt{b_{-}}<1$, if not the roles of $x_{ \pm}^{\mathrm{s}}$ are simply reversed in the following.) However, for $\epsilon>\epsilon_{\mathrm{c}}$, where $\epsilon_{\mathrm{c}}$ is such that

$$
\begin{gathered}
\frac{a_{+}}{b_{+}}\left(1-\frac{\sqrt{b_{+} / \epsilon_{\mathrm{c}} D} x_{+}^{\mathrm{s}, \max }}{\sinh \left(\sqrt{b_{+} / \epsilon_{\mathrm{c}} D} x_{+}^{s, \max }\right)}\right)+\frac{a_{-}}{b_{-}} \\
<\frac{2 \nu^{\prime}(0) \sqrt{b_{+} \epsilon_{\mathrm{c}} D}}{\tanh \left(\frac{1}{2} \sqrt{b_{+} / \epsilon_{\mathrm{c}} D} x_{+}^{s, \max }\right)}\left(1+\frac{a_{-}}{a_{+}}\right)
\end{gathered}
$$

the LHS of (2.14) is smaller than the RHS for all $x_{+}^{\mathrm{s}}, 0 \leq x_{+}^{\mathrm{s}} \leq x_{+}^{\mathrm{s}, \max }$, and no stall solution exists. In fact, for $\epsilon>\epsilon_{\mathrm{c}}$, no solution exists in a range $0 \leq c$ $<c_{\text {min }}(\epsilon)$, where $c_{\text {min }}$ is an increasing function of $\epsilon$.

The picture that emerges, for $\epsilon$ fixed and small, is then as follows. At the largest velocities, $x_{ \pm}$are large, $O\left(\epsilon^{-1 / 2}\right)$ and are well approximated by the diffusionless limit (2.8). As $c$ decreases, $x_{ \pm}$decrease. Significant departures from the diffusion- less limit appear when $c \approx \epsilon^{1 / 3}$. Here, the behavior of $x_{ \pm}$is governed by the Fife results, with a rate of decrease of $x_{ \pm}$much slower than the approximate quadratic relationship $x_{ \pm} \propto c^{2}$ of the diffusionless limit. Eventually, for smaller velocities, $c \ll \epsilon^{1 / 3}$, the Fife approximation does worse. The situation is now controlled by the stall limit. If $\epsilon \leq \epsilon_{\mathrm{c}}$, so that the stall limit exists, then $x_{ \pm}$approach their stall limits, $x_{ \pm}^{s}$. In general, $x_{+}$always decreases with $c$, whereas $x_{-}$can have a turning point if $\epsilon$ is insufficiently small. If $\epsilon>\epsilon_{\mathrm{c}}$, there is a velocity at which $x_{-}, \infty$, below which no periodic traveling wave solution exists.

We can verify this picture by numerically solving the system of equations (2.7). For these calculations, we fix (for no particular reason) $D=b_{+}=$ $b_{-}=1, a_{+}=0.625, a_{-}=0.375$. We also assume for $\nu$, the function relating $c$ to the value of $v$ on the interface: $\nu(c)=c / 2 \sqrt{c^{2}+4}$, which is the form that arises from a piecewise-linear dynamics for $u$. The results of the calculations are presented in figs. 3-5. In figs. $3 a$ and $3 b$, we plot $x_{ \pm}$as a function of $c$ for $\epsilon=0.01$, below the critical value $\epsilon_{\mathrm{c}} \approx 0.163$ for this choice of parameters. Also presented on the same graphs are the results of the diffusionless and Fife approximations. One way to measure the quality of the Fife approximation is to consider $x_{-} / x_{+}$. In the Fife approximation, this ratio is a constant, $a_{+} / a_{-}=5 / 3$, independent of $c$. This ratio is plotted in fig. 4 as a function of $\tilde{c}$, where we see that $x_{-} / x_{+}$approaches the Fife value as $\tilde{c}$ decreases from its maximum value. It comes closest at $\tilde{c} \approx 0.495$, where $x_{-} / x_{+} \approx 1.763$, and then diverges from the Fife result again from smaller $\tilde{c}$. In fig. 4, we also present $x_{-} / x_{+}$versus $\tilde{c}$ for a smaller valuc of $\epsilon=10^{-3}$. For this smaller value of $\epsilon$, the Fife approximation does better, as expected. For example, $x_{-} / x_{+}$now goes down to 1.693, compared to the Fife value of 1.667. Lastly, we vary $a_{ \pm}$, so that $\epsilon$ is above $\epsilon_{\mathrm{c}}$, while still small. Setting $a_{+}=0.8, a_{-}=0.2$, our original choice of $\epsilon=0.01$ is now above the critical value, $\epsilon_{\mathrm{c}} \approx 8.73$ $\times 10^{-3}$, for these parameters. In figs. $5 \mathrm{a}$ and $5 \mathrm{~b}$ we plot $x_{ \pm}$versus $c$, together with the Fife prediction. We see that while the results for $x_{+}$are 


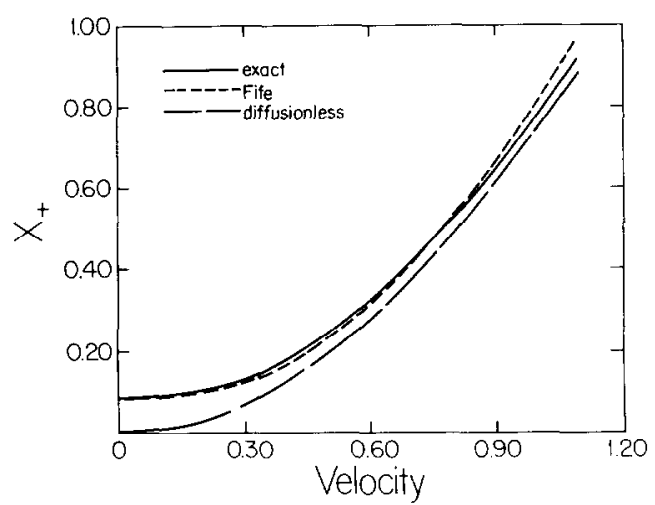

(a)

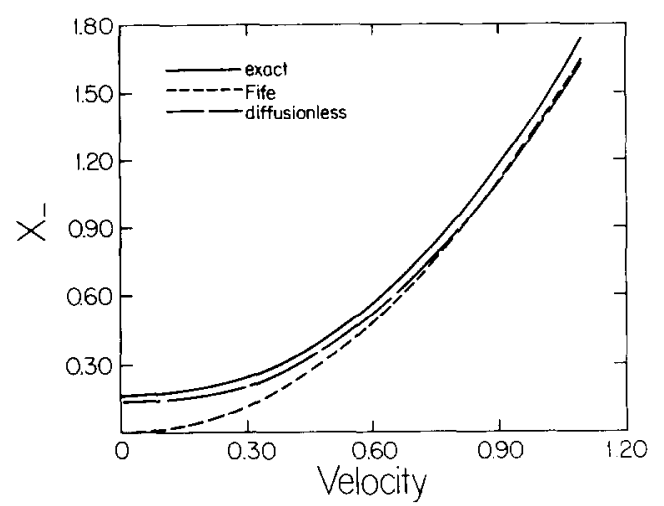

(b)

Fig. 3. $x_{+}$(a) and $x_{-}$(b) versus velocity for the PLM with parameters $\epsilon=0.01, D=f_{+}=f_{-}=1, g_{+}=0.625, g_{-}=0.375$. The solid curve is the exact results, which are plotted together with the results of the diffusionless approximation (long dashes) and the Fife approximation (short dashes).

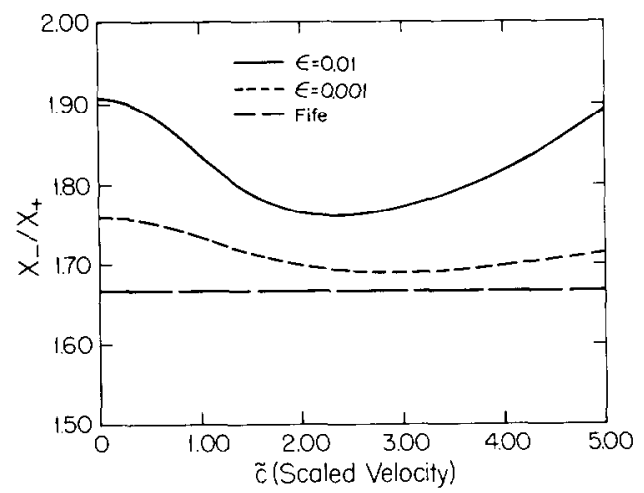

Fig. 4. $x_{-} / x_{+}$versus the scaled velocity, $\tilde{c}$, for the PLM with $\epsilon=0.01$ (solid), 0.001 (short dashes) compared to the Fife prediction (long dashes). The other parameters are as in fig. 1.

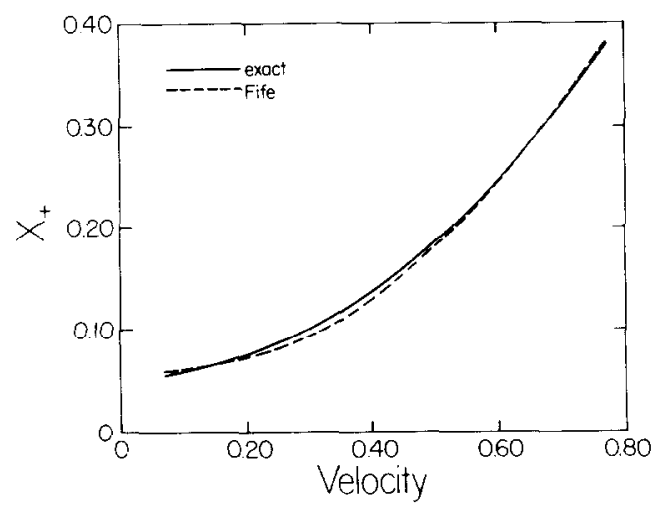

(a)

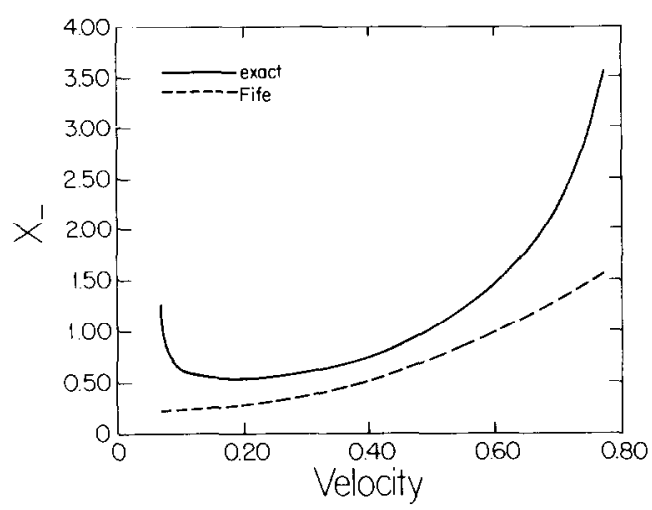

(b)

Fig. 5. $x_{+}$(a) and $x_{-}$(b) versus velocity for the PLM with parameters $\epsilon=0.01, D=f_{+}=f_{-}=1, g_{+}=0.8, g_{-}=0.2$. Exact results (solid) and Fife approximation (short dashes).

still fairly good, the results for $x_{-}$are completely unreliable, due to the divergence of $x_{-}$at the critical velocity, $c_{\min } \approx 0.0672$.

Our last task here is to calculate $\epsilon_{c}$, which controls the validity of the Fife approximation for small $c$. It is clear from the above that the most relevant parameter determining $\epsilon_{\mathrm{c}}$ is $a_{-} / a_{+}$. Let us then calculate $\epsilon_{\mathrm{c}}$ in the limit $a_{-} / a_{+} \ll 1$. In this limit, $x_{+}^{\mathrm{s}, \max } \approx 2 \sqrt{\epsilon D / b_{-}} a_{-} / a_{+}$, and so (2.15) reduces to

$\epsilon_{\mathrm{c}} \approx \frac{a_{-}^{4}}{4 a_{+}^{2} b_{-}^{3} D \nu^{\prime}(0)^{2}}$.

The Fife approximation is then valid at small 
velocity for $\epsilon \ll \epsilon_{\mathrm{c}}$. As we noted in the introduction to this subsection, this condition is much more stringent than our overall assumption $\epsilon \ll 1$.

\section{Spiral waves}

We will now examine the implications of the results of the previous section for the structure of spiral waves in BZ-type systems. Our analysis will be at first in the context of the PLM of section 2. We will see in the next section how the discussion can be generalized to more realistic models and in particular the Oregonator.

As pointed out in the introduction, the spiral wave pattern at large distances from the spiral core asymptotically approaches a periodic traveling wave solution of the type studied in section 2 . We have seen that such solutions exist for a continuous range of velocities, with the spatial periodicity determined by the velocity. The solution to the problem of velocity selection therefore lies in an understanding of the dynamics in the core region. In particular, one needs to determine the mechanism that sets the size of the core.

It is clear from the results of the previous section that diffusion of $v$ plays a crucial role in the core region, where the normal velocity of the interface is small. Indeed, this observation served as the motivation for the current study. We have seen that for small $\epsilon$, there is a natural length scale, $O\left(\epsilon^{2 / 3}\right)$, for velocities small enough that diffusion is important. This suggests that the length scale of the core of $\mathrm{O}\left(\epsilon^{2 / 3}\right)$.

We must now consider the length scale far from the core, where we have a periodic traveling wave solution. We derive in section 2 that there are two possibilities: either $c \gg \epsilon^{1 / 3}$ and diffusion is unimportant asymptotically; or $c \leqslant \epsilon^{1 / 3}$ and diffusion is relevant even in the far region. Of these, the latter is clearly the most attractive, as it would imply that the length scale in the outer region is also $O\left(\epsilon^{2 / 3}\right)$ and the entire pattern scales uniformly in $\epsilon$. If this is indeed the case, there is then the possibility of eliminating $\epsilon$ from the problem en- tirely through a rescaling. Of course, we do not know at this stage which scenario is correct, for this is precisely the problem of velocity selection we wish to eventually address, but for the moment let us assume the second scenario is realized and investigate the consequences.

Before proceeding, however, we must address the effects of interfacial curvature. As is well known $[6,11,14]$, the decoupling of the $u$ and $v$ equations in the small $\epsilon$ limit, which introduces a sharp interface between phases in the equation for $v$, is modified if the interface is curved. The effect of a nonzero interfacial curvature, $\kappa$, is to modify the $c-v_{0}$ relation [2.6] to

$v_{0}=\nu(c+\epsilon \kappa)$.

Now, we have already assumed that the length scale of the spiral is $O\left(\epsilon^{2 / 3}\right)$ everywhere, so $\kappa \sim$ $O\left(\epsilon^{-2 / 3}\right)$. If we are to have uniformity in $\epsilon$, it must also be the case that $c \sim O\left(\epsilon^{1 / 3}\right)$. These scalings, $x \sim O\left(\epsilon^{2 / 3}\right), c \sim O\left(\epsilon^{1 / 3}\right)$ are just those of the Fife regime.

We can now perform the rescalings and exhibit the $\epsilon$-independent equations, first derived by Fife [11]. Going to polar coordinates $(r, \theta)$ in the corotating frame with angular velocity $\omega$, we rescale $r \equiv \tilde{r} \epsilon^{2 / 3}, \omega \equiv \tilde{\omega} \epsilon^{-1 / 3}$, yielding

$$
\begin{aligned}
& \epsilon^{-1 / 3}\left[D\left(\frac{\partial^{2}}{\partial \tilde{r}^{2}}+\frac{1}{\tilde{r}} \frac{\partial}{\partial \tilde{r}}+\frac{1}{\tilde{r}^{2}} \frac{\partial^{2}}{\partial \theta^{2}}\right)+\tilde{\omega} \frac{\partial}{\partial \bar{\theta}}\right] v \\
& \pm a_{ \pm}-b_{ \pm}\left(v-v_{\mathrm{s}}\right)=0
\end{aligned}
$$

If we now rescale $v$, defining $\tilde{v} \equiv \epsilon^{-1 / 3}\left(v-v_{\mathrm{s}}\right)$, we get the final result

$\left(D \tilde{\nabla}^{2}+\tilde{\omega} \frac{\partial}{\partial \theta}\right) \tilde{v} \pm a_{ \pm}=0$,

where we have dropped the term $b_{+} \tilde{v} \epsilon^{1 / 3}$. This is valid as long as $v-v_{\mathrm{s}} \ll 1$. We saw in section 2 that this was true for the traveling wave solutions in the Fife regime, and it is reasonable to expect it to be true for the entire spiral wave, given that the pattern in the far regime is a Fife-regime travelling 
wave, as we are assuming. Eq. (3.3) has to be supplemented by the boundary condition for $\tilde{v}$ on the interphase boundary,

$\left.\tilde{v}\right|_{\text {interface }}=\nu^{\prime}(0)\left(\tilde{c}_{\mathrm{n}}+\tilde{\kappa}\right)$,

where $\tilde{c}_{n}$ is the (rescaled) normal velocity of the interface, and $\tilde{\kappa}$ the rescaled curvature.

We have thus rederived Fife's $\epsilon$-independent equations for a steady-state spiral. It of course remains to be seen what the nature of the solution space for these equations is. It is tempting to speculate, by analogy with the pattern selection seen in dendritic growth and viscous fingering, that there are a discrete infinity of steady-state solutions, with a unique stable solution. In the next section, we turn to the fully nonlinear Oregonator model, and investigate the implications of the Fife scaling hypothesis in this case.

\section{The Oregonator}

The PLM, while possessing the advantage of simplicity is at best only an approximation to a realistic model for the $B Z$ reaction. As we discussed in the introduction, the complication of a more exact model is that, even when the "fast" fields are decoupled, it involves a nonlinear field equation for the "slow" field, $v$. In particular, a reduction to interface dynamics is not possible.

If we assume, however, that the Fife scaling is appropriate to describe spiral wave patterns, a tremendous simplification results. We saw that one of the features of the Fife regime is that $v-v_{\mathrm{s}} \ll 1$ everywhere. This allows us to linearize the $v$ equation around the stall value $v_{\mathrm{s}}$. The result is a version of the PLM, with specific values of the parameters determined from the linearization. This is noteworthy for a number of reasons. First, the problem is now linear, allowing an attack via the same boundary-integral techniques used successfully in the dendrite and viscous fingering problems. The formulation of this approach will be the subject of the next section. Second, and most importantly, the structure of the resulting problem is independent of the fine details of the underlying Oregonator model. All that remains of this fine structure are the couplings $a_{ \pm}$(as we saw above, $b_{+}$is irrelevant in the Fife region). These couplings can be determined phenomenologically from the dispersion relation for plane travelling waves. Thus, given a solution of the Fife-reduced PLM spiral wave problem ((3.3) and (3.4)) one can directly make predictions for the realistic case without making use of the detailed structure of the original model. This is very attractive, as the "realistic" models discussed in the literature, including the Oregonator, are themselves only phenomenological models, and are surely not correct in all details.

This situation is reminiscent of that encountered in the dendrite problem. There, one typically studies a "macroscopic" model, in which the interface is approximated as being infinitely thin. One can choose to start, however, from a phasefield approach [14], where one writes down a Landau-Ginzburg model for the dynamics of the rapidly varying phase field which gives rise to the interface. One can show [14] that in the limit of an infinitely thin interface, which in this case is obtained by letting the diffusion constant of the phase field tend to zero, one obtains the macroscopic model. This macroscopic model is insensitive to the fine structure of the Landau-Ginzburg theory, which just serves to determine the macroscopic couplings (e.g. the surface tension). These couplings can in turn be directly obtained from experiments.

This wonderfully attractive scenario depends crucially on two things. First is the question in principle of whether the Fife scaling is correct and gives rise to a unique stable steady state. This question has already been discussed at length in section 3 , and its resolution must await further work. The other question is a more practical matter concerning the smallness of $\epsilon$. The Fife scaling is predicated on the $\epsilon \rightarrow 0$ limit (or in other words, the infinitely thin interface limit). We saw in section 2 how the Fife regime can break down at 
small velocities if $\epsilon$ is not small enough. While we would not expect qualitative changes in the physics to occur as long as $\epsilon$ is reasonably small (for example, if the Fife scalings produce a unique stable spiral one would expect this feature to persist for finite $\epsilon$ ), the quantitative predictions will become worse as $\epsilon$ grows. One way to get a feel for this question is to return to the problem of periodic travelling waves studied in section 2 .

This question of course depends on the particular chemistry one is studying. Let us focus for the sake of definiteness on the Oregonator [15] model:

$\epsilon \dot{u}=\epsilon^{2} \nabla^{2} u+f(u, v)$,

$\dot{v}=\epsilon \nabla^{2} v+g(u, v)$,

where the reaction terms $f, g$ are given by

$f(u, v)=u-u^{2}-\hat{f v} \frac{u-q}{u+q}$,

$g(u, v)=u-v$

The couplings are given by $\hat{f} \approx 3, q \approx 10^{-4}$, and, most importantly, $\epsilon \approx 10^{-2}$. The linearization of this model has been performed numerically by DKT. Here we will exploit the smallness of $q$ to exhibit (approximate) analytical results. As usual, the smallness of $\epsilon$ implies a two-phase structure with a sharp interface separating the two phases. In the "+" phase,

$u_{+}(v) \approx \frac{1}{2}(1+\sqrt{1-4 \hat{f v}})$

and in the "-" phase

$u_{-}(v) \approx q+\frac{2}{\hat{f v}} q^{2}$.

As advertised, substituting $u(v)$ in (4.1b), we find that $v$ satisfies a nonlinear field equation.

To linearize around the stall solution, we need first compute $v_{\mathrm{s}}$. The easiest way to do this is to consider $F_{v}(u)$, where $f(u, v) \equiv \partial F_{v} / \partial u$. $F_{v}$ has two local maxima, $u_{ \pm}(v)$, and $v_{\mathrm{s}}$ is determined by the condition $F_{v^{\prime}}\left(u_{+}\left(v_{\mathrm{s}}\right)\right)=F_{v_{\mathrm{s}}}\left(u_{-}\left(v_{\mathrm{s}}\right)\right)$. To lowest order in $q$, we find $v_{\mathrm{s}} \approx 3 / 16 \hat{f}$. We can now expand (4.1b) to linear order, obtaining

$$
\begin{aligned}
\dot{v}= & \nabla^{2} v+g\left(u_{ \pm}\left(v_{\mathrm{s}}\right), v_{\mathrm{s}}\right) \\
& +\left.\left(\frac{\partial g}{\partial u} \frac{\partial u_{ \pm}}{\partial v}+\frac{\partial g}{\partial v}\right)\right|_{u=u_{+}\left(v_{\mathrm{s}}\right) ; v=v_{\mathrm{s}}}\left(v-v_{\mathrm{s}}\right) .
\end{aligned}
$$

This is just the equation of motion for $v$ in the PLM with $D=1$ and the identifications

$$
\begin{aligned}
a_{ \pm} & = \pm g\left(u_{ \pm}\left(v_{\mathrm{s}}\right), v_{\mathrm{s}}\right) \\
& \approx 3 / 4-3 / 16 \hat{f,}, "+\cdots, \\
& \approx 3 / 16 \hat{f}, \quad \cdots-"
\end{aligned}
$$

and

$$
\begin{aligned}
b_{ \pm} & =-\left.\left(\frac{\partial g}{\partial u} \frac{\partial u_{ \pm}}{\partial v}+\frac{\partial g}{\partial v}\right)\right|_{u=u_{ \pm}\left(v_{\mathrm{s}}\right) ; v=v_{\mathrm{s}}} \\
& \approx 2 \hat{f}+1, \quad “+" \\
& \approx 1,
\end{aligned}
$$

We also need the relation between the normal velocity $c$ of the interface and the value of $v$ on the interface. Neglecting curvature, (4.1a) implics

$\epsilon c \int_{-\infty}^{\infty} \mathrm{d} x^{\prime}\left[u^{\prime}\left(x^{\prime}\right)\right]^{2}=F_{v}\left(u_{-}\right)-F_{v}\left(u_{+}\right)$,

where $u(x)$ is the kink solution connecting $u_{-}(v)$ at $x=-\infty$ to $u_{+}(v)$ at $x=\infty$. For small velocities, $u(x) \approx u_{\mathrm{s}}(x)$ the zero-velocity kink solution. Then,

$$
\begin{aligned}
& \epsilon c \int_{-\infty}^{\infty} \mathrm{d} x^{\prime}\left[u^{\prime}\left(x^{\prime}\right)\right]^{2} \approx \epsilon c \int_{u_{-}\left(v_{\mathrm{s}}\right)}^{u_{+}\left(v_{\mathrm{s}}\right)} \mathrm{d} u \frac{\mathrm{d} u_{\mathrm{s}}}{\mathrm{d} x} \\
& \quad=c \int_{u_{-}\left(v_{\mathrm{s}}\right)}^{u_{+}\left(v_{\mathrm{s}}\right)} \mathrm{d} u \sqrt{2\left[F_{u_{s}}\left(u_{-}\left(v_{\mathrm{s}}\right)\right)-F_{v_{\mathrm{s}}}(u)\right]} \\
& \quad \approx \frac{3 \sqrt{2}}{40} c
\end{aligned}
$$

again to lowest order in $q$. After expanding the 
RHS of (4.6) about $v=v_{s}$, we obtain

$v \approx v_{\mathrm{s}}+\frac{\sqrt{2}}{10 \hat{f}} c$

In the notation of section $2, \nu^{\prime}(0) \approx \sqrt{2} / 10 \hat{f}$.

In section 2, we saw that $\epsilon_{\mathrm{c}}$, the value of $\epsilon$ above which the $c=0$ traveling wave solution ceased to exist, is a good figure of merit for the border between the very small $\epsilon$ regime where the Fife results are trustworthy, and the regime where $\epsilon$, though small, is too large for the Fife approximation to be useful. We may obtain an estimate for $\epsilon_{c}$ from our linearized version of the Oregonator (the piecewise linear Oregonator (PLO)), embodied in (4.4) and (4.5). We remind the reader that the Fife limit is obtained from the PLO by dropping the $v-v_{\mathrm{s}}$ term. While the linearization by means of which we derived the PLO is strictly speaking only valid in the Fife limit, we saw in section 2 that the breakdown of the Fife limit at small velocity for $\epsilon$ not small enough is caused by precisely the $v-v_{\mathrm{s}}$ term. It is thus reasonable to use the PLO to obtain an estimate for $\epsilon_{\mathrm{c}}$. From (2.15), along with the values of $a_{ \pm}, b_{ \pm}$given in (4.5), we find $\epsilon_{\mathrm{c}}=3.48 \times 10^{-3}$. Thus, $\epsilon$ for the Oregonator as given by Keener and Tyson is in fact much larger than $\epsilon_{\mathrm{c}}$. We should therefore expect that the comparison to experiment of numerical predictions based on the Fife limit should not be terribly accurate.

One can verify this further by comparing the results of the Oregonator for the wavelength of the periodic traveling wave train both to the Fife limit and to the PLO. This is done in figs. $6 \mathrm{a}$ and $6 \mathrm{~b}$, which present $x_{+}$and $x_{-}$versus $c$ for the three models. We see that there $x_{-} \rightarrow \infty$ at $c \approx 2.57$ (as against $c \approx 2.502$ in the PLO) below which there is no solution. As expected, the Fife approximation does a poor job, especially in predicting $x_{-}$. The PLO, however, does surprisingly well over the whole range of velocities for which the Oregonator has travelling wave train solutions, as already noted by DKT from a similar comparison. It should be reiterated that this is indeed a happy

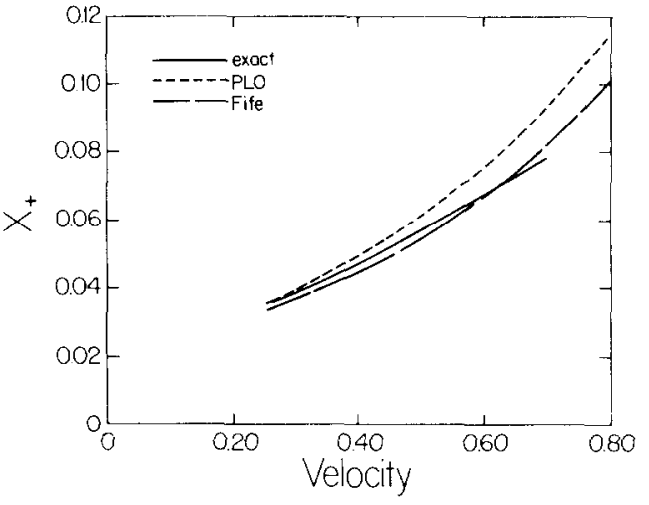

(a)

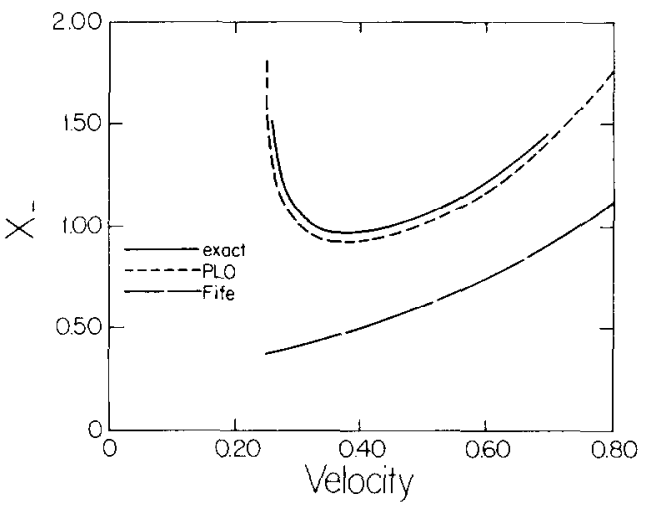

(b)

Fig. 6. $x_{+}$(a) and $x$. (b) versus velocity and the Oregonator (solid) compared to the piecewise-linear Oregonator (short dashes) and the Fife approximation (long dashes).

numerical accident, stemming from the fact that both the velocity relation $v_{0}(c)$ and $u_{*}(v)$ are very close to linear over the relevant range of their arguments. The lesson here is that the PLO might do a reasonable quantitative job in predicting spiral patterns, even though $\epsilon$ is too large for the Fife regime to work well.

\section{The boundary integral formulation}

The important advantage of the PLM is that one can hope to attack this problem for arbitrary $\epsilon$ due to the linearity of the model. In so doing, one can test the validity of the Fife scalings in the 
small $\epsilon$ limit. As discussed in the introduction, a reformulation in terms of a boundary-integral problem should prove most useful in this analysis. To accomplish this, we need first to define the appropriate Green's functions for the differential operator in (3.2):

$$
\begin{gathered}
\left(\tilde{\nabla}^{2}+\frac{\tilde{\omega}}{D} \frac{\partial}{\partial \theta}-\frac{\epsilon^{1 / 3}}{D} b_{ \pm}\right) G_{ \pm} \\
=\frac{1}{\tilde{r}} \delta\left(\tilde{r}-\tilde{r}^{\prime}\right) \delta\left(\theta-\theta^{\prime}\right) .
\end{gathered}
$$

The presence of different Green's functions in the two phases complicates the boundary-layer analysis somewhat. The problem can be treated [16] by introducing two fields, $\psi_{ \pm}$, each of which is nonzero only in its respective phase. We then define

$$
\begin{aligned}
\psi_{ \pm} & =v-v_{\mathrm{s}} \mp a_{ \pm} / b_{ \pm} & & \text {"correct" phase, } \\
& =0 & & \text { otherwise. }
\end{aligned}
$$

We can then write

$\psi_{ \pm}=\int \hat{n}^{\prime} \cdot \tilde{\nabla}^{\prime} G_{ \pm} \phi_{1}^{ \pm} \mathrm{d} \tilde{s}^{\prime}-\int G_{ \pm} \phi_{2}^{\frac{1}{2}} \mathrm{~d} \tilde{s}^{\prime}$

where the integrals are along the interface, and $\hat{n}$ is the normal to the interface, taken to point toward the "+" region. The functions $\phi_{1}, \phi_{2}$ are determined by the discontinuities of $\psi$ and $\hat{n} \cdot \tilde{\nabla} \psi$ across the interface:

$$
\begin{aligned}
& {[\psi]_{\mathrm{disc}}=\phi_{1},} \\
& {[\hat{n} \cdot \tilde{\nabla} \psi]_{\mathrm{disc}}=\phi_{2}-\frac{\tilde{c}_{\mathrm{n}}}{D} \phi_{1},}
\end{aligned}
$$

where the discontinuity is calculated from the " $-"$ to the "+" phase. The continuity of $v$ and $\hat{n} \cdot \tilde{\nabla} v$ across the interface, together with the boundary condition (3.1), implies that

$$
\begin{aligned}
& \phi_{1}^{ \pm}= \pm\left[\nu\left(\epsilon^{1 / 3}\left(\tilde{c}_{\mathrm{n}}+\tilde{\kappa}\right)\right) \quad v_{\mathrm{s}} \uparrow a_{ \pm} / b_{ \pm}\right], \\
& \phi_{2}^{ \pm}= \pm \phi+\frac{\tilde{c}_{\mathrm{n}}}{D} \phi_{1}^{ \pm},
\end{aligned}
$$

where $\phi$ is the (unknown) value of $\hat{n} \cdot \nabla v$ at the interface. Using this, we can now evaluate (5.5) on the "wrong" side of the interface, where $\psi$ is zero. These two equations at each point on the interface together determine the interface, along with the unknown function $\phi$.

Things simplify somewhat in the Fife limit. Here we only need one Green's function, satisfying

$\left(\tilde{\nabla}^{2}+\frac{\tilde{\omega}}{\bar{D}} \frac{\partial}{\partial \theta}\right) G=\frac{1}{\tilde{r}} \delta\left(\tilde{r}-\tilde{r}^{\prime}\right) \delta\left(\theta-\theta^{\prime}\right)$,

so the boundary-integral equations can be formulated in terms of one field, $\psi$, define by subtracting the inhomogeneous solution to (3.3) from $\tilde{v}$,

$\psi=\tilde{v} \pm \frac{\tilde{r}^{2}}{4 D} a_{+}$

As in (5.5), (upon dropping the \pm 's), $\psi$ can be expressed as an integral of the Green's function along the interface. The source strengths are now given by

$\phi_{1}=\frac{\tilde{r}^{2}}{4 D}\left(a_{+}+a_{-}\right)$,

$\phi_{2}=\frac{\tilde{r}}{2 D}\left(a_{+}+a_{-}\right) \hat{n} \cdot \hat{r}+\frac{\tilde{c}_{\mathrm{n}}}{D} \phi_{1}$.

As before, we now evaluate $\psi$ along the interface using (5.5), applying the boundary condition (3.4). The resulting equation determines the interface shape.

\section{Summary}

In summary, then, we have reviewed the structure of traveling wave solutions to the PLM. We characterized the three different velocity regimes and the corresponding length scales: (1) the large velocity region where $c, x_{ \pm} \sim \mathrm{O}(1)$ and diffusion is irrelevant; (2) the Fife regime, which sets in for $c \sim \mathrm{O}\left(\epsilon^{1 / 3}\right)$, where diffusion becomes important and $x_{ \pm} \sim O\left(\epsilon^{2 / 3}\right)$; and (3) the small-velocity re- 
gion, where the behavior is very sensitive to the value of $\epsilon$ and differs dramatically from the Fife results if $\epsilon$ is too large.

We then demonstrated that assuming the selected velocity of the spiral pattern lies in the diffusion-dominated Fife regime (and indeed assuming there is a selected velocity in the first place), we can reproduce the $\epsilon$-independent equations of Fife. It is illuminating to note in this context that the actual experimental value for the spiral velocity does indeed appear to lie in the regime where diffusion effects are important. This can be seen clearly by comparing figs. $3 a$ and 8 of Keener and Tyson [6]. As a first step toward addressing the validity of this assumption, we have formulated a boundary-integral representation of the steady-state spiral equations, both with the assumption of Fife scaling and also in general.

Lastly, we pointed out that the Fife equations are in fact universal, independent of the details of the underlying equations. The underlying dynamics serves only to fix the coupling constants in the Fife equation. This derivation relies on taking the $\epsilon \rightarrow 0$ limit, in which there is an infinitely sharp interface between the two "phases" of the $v$ field. It would thus be very useful to have experimental data on the $\epsilon$ dependence of the spiral pattern. which can presumably be obtained through vary ing the recipe used in the reaction. This is especially true since the value of $\epsilon$ for the particular recipe analyzed by Keener and Tyson is not small enough for the Fife theory to be quantitatively correct. The piecewise-linear Oregonator, a linearized version of the Oregonator, might, however, suffice for quantitative predictions for the Keener-Tyson recipe.

\section{Acknowledgements}

The work of D.A.K. was supported by U.S. Department of Energy, Grant No. DE-FG-0285ER54189. H.L. was supported in part by a grant from DARPA under the University Research Initiative Grant No. N00014-86-K-0758, and by the Alfred Sloan Foundation.

\section{References}

[1] R.J. Field and M. Burger, eds., Oscillations and Travelling Waves in Chomical Systems (Wiley, New York. 1985).

[2] S.L. Muller, T. Plesser and B. Hess, Science 230 (1985) 661 ;

W.Y. Tam, W. Horsthemke, Z. Noszticzius and H. Swinney, J. Chem. Phys. 88 (1988) 3395.

[3] J. Rinzel and J.B. Keller, Biophys. J. 13 (1973) 1313.

[4] J. J. Tyson and P. Fifc, J. Chem. Phys. 73 (1980) 2224.

[5] A.T. Winfree, Oscillations and Travelling Waves in Chemical Systems, R.J. Field and M. Burger, eds. (Wiley, New York, 1985).

[6] J.P. Keener and J.J. Tyson, Physica D 21 (1986) 307 ; J.P. Keener, SIAM J. Appl. Math. 46 (1986) 1039.

[7] M.E. Glicksman, Mat. Sci. Eng. 65 (1984) 45; A. Dougherty and J.P. Gollub, Phys. Rev. A 38 (1988) 3043 .

[8] P.G. Saffman and G.I. Taylor, Proc. Roy. Soc. A 245 (1958) 312.

[9] For a review of recent progress, see D. Kessler, J, Koplik and $H$. Levine, Pattern selection in fingered growth phenomena, Adv. Phys. 37 (1988) 255:

J.S. Langer, Les Houches Lecture Notes "On Chance and Matter". 1986, J. Souletic, J. Vannimenus and R. Stora, eds. (North-Holland, Amsterdam, 1987).

[10] J.D. Dockery, J.P. Keener and J.J. Tyson, Physica D 30 (1988) 177 .

[11] P. Fife, in: Non-equilibrium Dynamics in Chemical Systems, C. Vidal and A. Pacault, eds. (Springer, Berlin (1984); J. Stat. Phys. 39 (1985) 687.

[12] G. Nash and M.E. Gicksman, Acta Metall. 22 (1974) 1283;

D.A. Kessler and H. Levine, Phys. Fluids 30 (1987) 1246.

[13] Sec, e.g. H.P. McKean, Adv. Math. 4 (1975) 209.

[14] G. Fix, in: Research Notes in Mathematics, Vol. 2, A. Fanans and M. Primicero, eds. (Pitman, London, 1985); J. Collins and H. Levine, Phys. Rev. B31 (1985) 6119.

[15] J.J. Tyson, in: Oscillations and Travelling Waves in Chemical Systems. R.J. Field and M. Burger, eds. (Wiley, New York. 1985): and also in: Non-linear Phenomena in Chemical Dynamics, C. Vidal and A. Pacault, eds. (Springer, Berlin. 1981).

[16] D.A. Kessler and H. Levine, Steady-state cellular growth during direction solidification, Phys. Rev. A., to appear. 